\title{
X-ray absorption toward the red quasar 3C 212
}

\section{Citation}

Elvis, Martin, Fabrizio Fiore, Smita Mathur, and Belinda J. Wilkes. 1994. "X-Ray Absorption

Toward the Red Quasar 3C 212." The Astrophysical Journal 425 (April): 103. doi:10.1086/173966.

\section{Published Version}

doi:10.1086/173966

\section{Permanent link}

http://nrs.harvard.edu/urn-3:HUL.InstRepos:30212139

\section{Terms of Use}

This article was downloaded from Harvard University's DASH repository, and is made available under the terms and conditions applicable to Other Posted Material, as set forth at http:// nrs.harvard.edu/urn-3:HUL.InstRepos:dash.current.terms-of-use\#LAA

\section{Share Your Story}

The Harvard community has made this article openly available.

Please share how this access benefits you. Submit a story.

\section{Accessibility}


The ASTROPHYSICAL Journal, 425:103-109, 1994 April 10

(C) 1994. The American Astronomical Society. All rights reserved. Printed in U.S.A.

\title{
X-RAY ABSORPTION TOWARD THE RED QUASAR 3C 212
}

\author{
Martin Elvis, ${ }^{1}$ Fabrizio Fiore, Smita Mathur, and Belinda J. Wilkes \\ Harvard-Smithsonian Center for Astrophysics, 60 Garden Street, Cambridge, MA 02138 \\ Received 1993 August 30; accepted 1993 October 12
}

\begin{abstract}
A ROSAT X-ray spectrum of the $z=1.049$ "red quasar" 3C 212 has a strong low-energy cutoff. The spectrum can be fitted with a power law (of energy index $1.4_{-0.6}^{+0.8}$ ) with low-energy photoelectric absorption in excess of the Galactic value that, if at the redshift of the quasar, would have a column density of $\left(0.9_{-0.6}^{+0.8}\right)$ $\times 10^{22}$ atoms $\mathrm{cm}^{-2}$. Possible sites for the absorption are a nuclear torus, an intervening damped Lyman- $\alpha$ system, or intracluster material (e.g., a cooling flow) around the quasar. The implied absorbing column density is sufficient to redden a normal quasar spectrum to the observed steep optical slope. The observed continuum, if dereddened by this amount, can produce the observed emission line fluxes and ratios. The absence of the graphite $\lambda 2175$ feature in 3C 212 however, requires dust different from the local Milky Way composition, or an intervening absorber with $z<0.4$.

Alternative acceptable fits to the X-ray spectrum are (1) a blackbody with a temperature of $0.7 \mathrm{keV}$ (in the quasar frame) modified only by Galactic absorption, and (2) an optically thin thermal plasma with excess absorption. Although a blackbody spectrum would be unprecedented, the model is consistent with all the available X-ray and optical data and cannot be ruled out. We discuss possible observations that can discriminate among the above models.
\end{abstract}

Subject headings: quasars: individual (3C 212) - X-rays: galaxies

\section{INTRODUCTION}

Around 1980 a number of "blank field" radio sources - that is, sources with very faint optical counterparts - were found to be bright near-infrared (1-2 $\mu \mathrm{m})$ emitters (Rieke et al. 1979, 1982). The large IR fluxes and faint optical limits implied that the spectra fell steeply toward the blue $\left(\alpha>3, f_{v} \propto v^{-\alpha}\right)$, hence these objects were dubbed "red quasars." Little is known about these objects, yet they are quite common, comprising $\sim 15 \%$ of the 3CR sample (Smith \& Spinrad 1980). While several of the "red quasars" are actually blazars (given their rapid variability and polarization, Bregman et al. 1981; Impey, Lawrence, \& Tapia 1991), other red quasars show broad emission lines of fairly normal rest equivalent width $[\mathrm{EW}(\mathrm{Mg}$ II $)=10-22 \AA$, Smith \& Spinrad 1980; cf. $27 \pm 15 \AA$ for normal radio-loud quasars, Wilkes 1986]. They have low optical polarization (Moore \& Stockman 1984), and are not core-dominated radio sources (Browne \& Murphy 1987). Thus these objects are unlikely to be dominated by a beamed BL Lac-type component. None of the quasars shows the $\lambda 2175$ dust absorption feature that would indicate a reddened spectrum (Smith \& Spinrad 1980). Instead these objects are quasars which appear to be devoid of the much-studied "UV bump" (e.g., Sun \& Malkan 1989).

The discovery of quasars with no "UV bump" would require a major reevaluation of quasar models (e.g., Eracleous \& Halpern 1994) and would give an opportunity to examine the effect of the "UV bump" on the emission of a quasar, both its X-ray spectrum and its emission lines. In pair plasma models the UV photons of the big bump can be upscattered by relativistic electrons to produce a power-law X-ray spectrum (e.g., Svensson 1990). In the absence of a soft photon source a

\footnotetext{
${ }^{1}$ E-mail: elvis@cfa.harvard.edu.
}

hard X-ray $/ \gamma$-ray spectrum results $\left(\alpha_{\mathrm{X}}<0\right.$, Zdziarski \& Lamb 1986; $\alpha_{\mathbf{X}}<0.5$, Done \& Fabian 1989). Also Smith \& Spinrad show that an extrapolation of the optical continuum does not produce enough ionizing photons to explain the strong $\mathrm{H} \beta$ line observed, leaving only X-rays as a source of photoionization. These properties make red quasars a natural target for X-ray spectral observations. Because "UV bump-free" quasars are potentially so interesting it is essential to be sure that reddening is not the cause of their optical colors, since the $\lambda 2175$ feature is not a trustworthy measure of obscuration (Savage \& Mathis 1979). A soft X-ray spectrum provides a good means of testing for intervening absorption.

We observed 3C 212, the brightest of the red quasars detected in X-rays by Bregman et al. (1985), with ROSAT in order to obtain a spectrum, and we report the results here. We use a Friedman cosmology with $H_{0}=50 \mathrm{~km} \mathrm{~s}^{-1} \mathrm{Mpc}^{-1}$ and $\Omega_{0}=1$ throughout. 3C 212 has a redshift of 1.049 (Smith \& Spinrad 1980). The Galactic H I column density toward 3C 212 is $3.7 \times 10^{20}$ atoms cm $\mathrm{cm}^{-2}$ (Stark et al. 1992). The uncertainty on this column density is $\sim 1 \times 10^{20}$ atoms cm $\mathrm{cm}^{-2}$ ( $90 \%$ confidence, Elvis et al. 1986).

\section{OBSERVATIONS AND DATA ANALYSIS}

The ROSAT Position Sensitive Proportional Counter (PSPC, Trümper 1983; Pfefferman et al. 1987) observed 3C 212 on 1992 May 12-18 for a total of 21,565 s (observation number rp700436). The quasar was centered in the field of view, and the "wobble" mode was on. The data were processed using the Standard Analysis Software System (SASS, version 5_7). An $\mathrm{X}$-ray source at the field center was detected with 770 net counts in PI channels $11-245(\approx 0.1-2.45 \mathrm{keV})$. Source counts were taken from within a circle of radius $2^{\prime}$. Such a circle contains $95 \%$ of the counts in a point source (Turner \& George 1992). The source counts in both the entire energy band and in the soft (PI $11-40 \approx 0.1-0.4 \mathrm{keV}$ ) energy band do not increase 
significantly when broader source regions are used. Background was extracted from an annulus of radii $3^{\prime}$ and $5^{\prime}$.

At these source flux levels $\left(\sim 5 \times 10^{-13}\right.$ ergs $\mathrm{cm}^{-2} \mathrm{~s}^{-1}$, $0.1-2.4 \mathrm{keV})$ confusing sources begin to make a significant contribution $(>15 \%)$ within our $2^{\prime}$ radius source circle. For this observation circles of $40^{\prime \prime}$ radius were removed from the source and background regions at six places where such sources were located (Fig. 1), two in the source region, and four in the background region. The extra sources within the 3C 212 source region lie 70" and 92" from 3C 212 and contain a total of 130 counts (within the $40^{\prime \prime}$ radius, $17 \%$ of $3 \mathrm{C} 212$ ), and the extra sources in the background region contain 275 counts, one third of the counts (828) in the final background region.

The centroid of the distribution is at R.A. $=08^{\mathrm{h}} 58^{\mathrm{m}} 41^{\mathrm{s}} .2$, decl. $=+14^{\circ} 09^{\prime} 34^{\prime \prime}(\mathrm{J} 2000.0)$, 11" from the optical position of 3C 212 (Smith \& Spinrad 1980) and well within the current positional uncertainties of ROSAT and the PSPC, leaving the identification of the X-ray source with the quasar in no doubt. The distribution of the X-rays from 3C 212 is a good fit to the PSPC point response function (FWHM $=25^{\prime \prime}$ calibrated using the radial distributions of 3C 273 and NAB $0205+024)$.

The PSPC count rate is 0.036 counts $\mathrm{s}^{-1}$, compared with the Einstein IPC count rate of 0.046 counts $\mathrm{s}^{-1}$. For a source with a spectrum with little low-energy flux, similar to that of $3 \mathrm{C} 212$ (see below), these correspond to roughly the same X-ray flux, so that no strong variation (greater than a factor 2) in X-ray flux is seen in the 12 years between the two observations.

It was immediately apparent from the pulse height distribution that the 3C $212 \mathrm{X}$-ray source contained virtually no photons below the $0.28 \mathrm{keV}$ carbon edge (Fig. 2), unlike the other, serendipitous, sources in the field. This obviously suggests low-energy photoelectric absorption above the Galactic value.

We made a number of spectral fits to the pulse height data employing the response matrix released on 1993 January (DRM_35_1). All spectral fits were made to the 34 PHA channels provided by the standard SASS analysis. Channels 1 and 2 were ignored as being less well calibrated (Turner \& George 1992), and channels with few counts were combined to ensure at least 10 counts per channel, so that $\chi^{2}$ statistics could be applied. We included in the analysis a standard flat systematic error of $1 \%$ as suggested by Hasinger \& Snowden (1992, private communication).

Our first fit used a simple power-law fit with absorption by cold material at zero redshift with solar abundances, and cross sections with Morrison \& McCammon (1983). Both the powerlaw slope and the absorbing column density were free to vary. The resulting value of $\chi^{2}$ is acceptable, but the best-fit absorb-

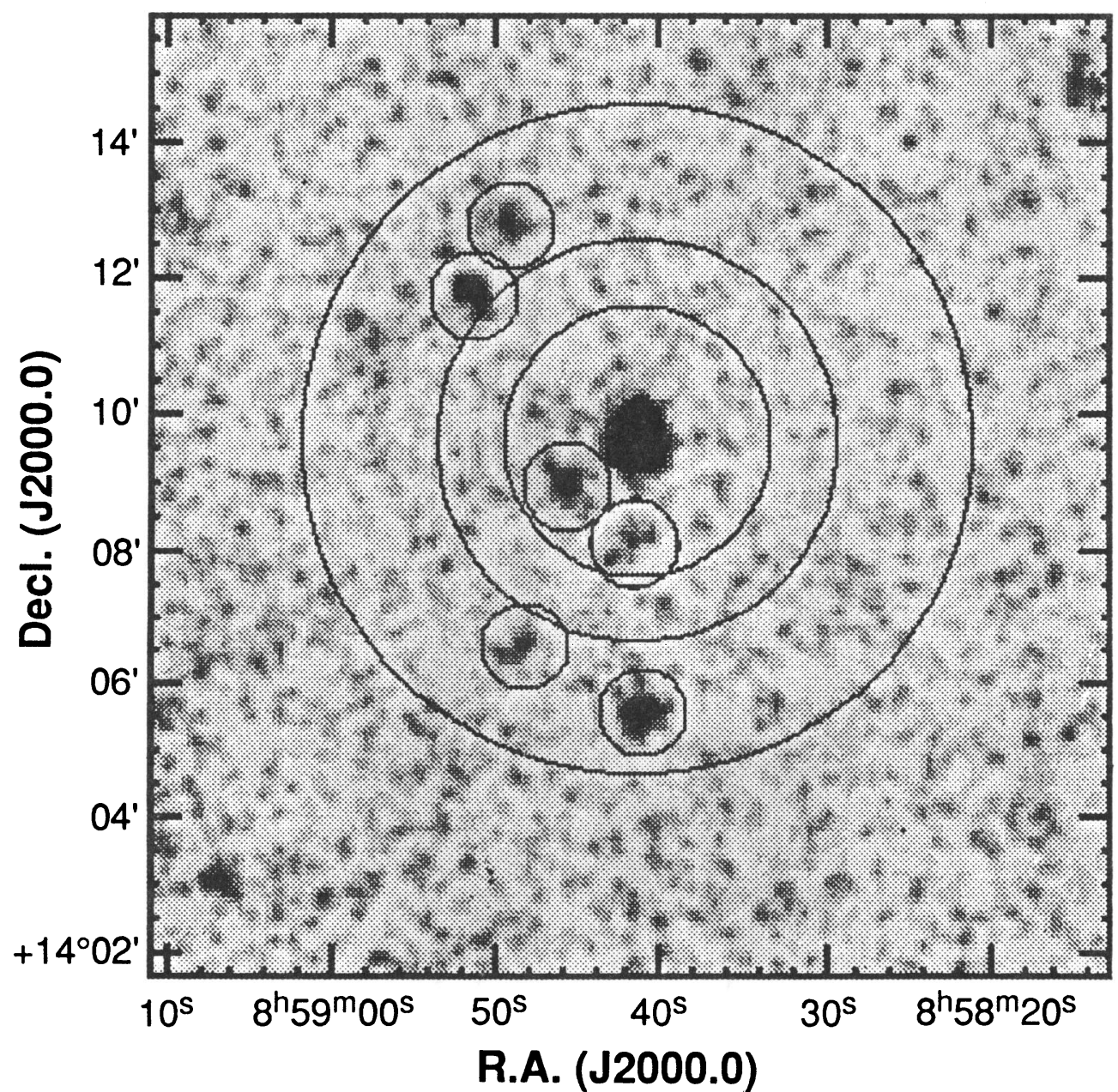

Fig. 1.-Central region of the ROSAT PSPC image centered on 3C 212. The greyscale image includes all pulse height channels and has been smoothed with a 5" sigma Gaussian. The three large circles centered on $3 \mathrm{C} 212$ have radii of $2^{\prime}, 3^{\prime}$, and $5^{\prime}$. Source counts were taken from the central $2^{\prime}$ circle; background counts were taken from the outer annulus between $3^{\prime}$ and $5^{\prime}$. The six small circles have radii of $40^{\prime \prime}$ and show the regions containing serendipitous confusing sources that were removed from the source and background regions. 3C 212 has a $0.2-2.4 \mathrm{keV}$ flux of $\sim 5 \times 10^{-13} \mathrm{ergs} \mathrm{cm}^{-2} \mathrm{~s}^{-1}$. The two sources within the source region have $17 \%$ of the net counts in $3 \mathrm{C} 212$. 


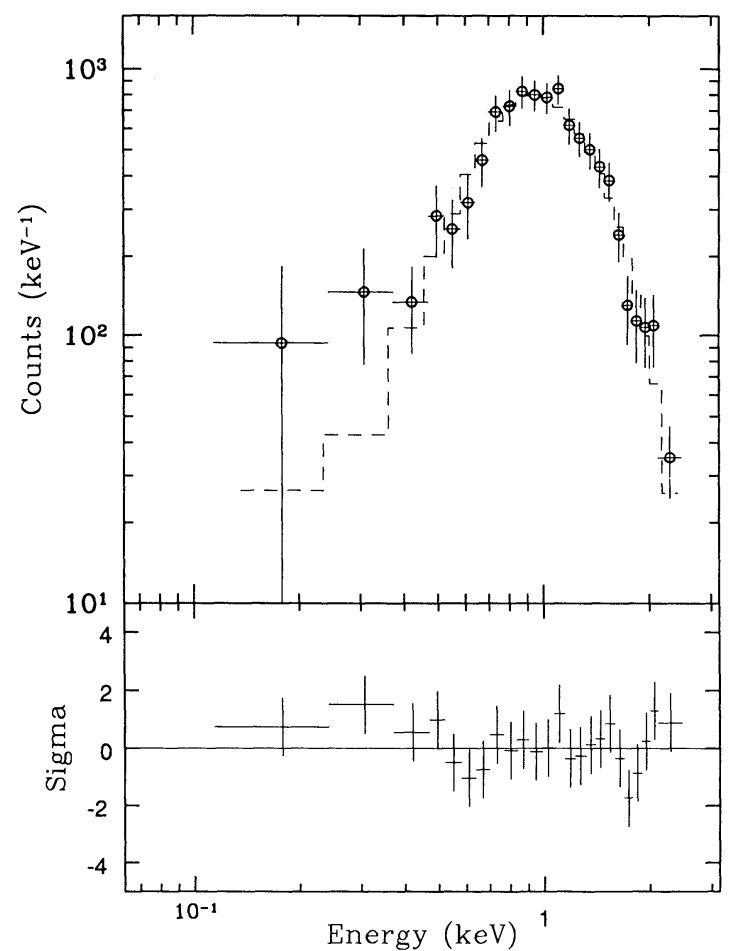

FIG. 2.-ROSAT PSPC pulse height spectrum of 3C 212 with the best-fit model of a power law with Galactic (fixed) and $z=1.049$ absorption (upper panel); residuals after the subtraction of the best-fitting power-law model (lower panel).

ing column density is an order of magnitude greater than the Galactic value. If a power-law fit is made with the Galactic column density (Table 1) then the increase in $\chi^{2}$ is unacceptable at above the $99.9 \%$ level $(F=23.2)$.

Excess absorption is clearly indicated, and an obvious site for this is within the quasar. We thus made another power-law fit with a $z=0$ absorption component fixed at the Galactic value plus another absorption component, which was allowed
TABLE 2

3C 212 X-RAY FluXes AND LUMINOSITIES

\begin{tabular}{cccc}
\hline \hline Model & $\begin{array}{c}f_{x}(0.2-2.4) \\
\mathrm{keV}^{\mathrm{a}}\end{array}$ & $\begin{array}{c}L_{x}(0.2-2.4) \\
\mathrm{keV}^{\mathrm{b}}\end{array}$ & $\begin{array}{c}L_{x}(0.4-4) \\
\mathrm{keV}^{\mathrm{b}}\end{array}$ \\
\hline Power law $\ldots \ldots \ldots \ldots \ldots \ldots$ & 4.3 & 8.9 & 7.4 \\
Blackbody $\ldots \ldots \ldots \ldots \ldots \ldots$ & 5.5 & 1.3 & 2.9 \\
Raymond thermal $\ldots \ldots \ldots$ & 4.4 & 5.4 & 5.4 \\
\hline
\end{tabular}

a $10^{-13} \mathrm{ergs} \mathrm{cm}^{-2} \mathrm{~s}^{-1}$, observed (i.e., no correction for absorption).

b $10^{45}$ ergs s$^{-1}$, corrected for absorption.

to vary, at the redshift of $3 \mathrm{C} 212$. This fit produced an acceptable $\chi^{2}$, and the fitted values are again listed in Table 1. Figure $3 a$ shows a contour plot of allowed values of the power-law energy index and $z=1.049$ absorbing column density. The implied column density at the quasar is increased by a factor of 3 over that of a $z=0$ absorber, since the energy of the photons being absorbed is higher by $(1+z)$, and is $\left(0.9_{-0.6}^{+0.8}\right) \times 10^{22}$ atoms $\mathrm{cm}^{-2}$. The power-law index is poorly constrained, at $1.4_{-0.6}^{+0.8}$.

Alternatives to absorbed power laws can also model the data well. A blackbody at the redshift of 3C 212 (or at redshift zero, with temperature decreased by a factor $1+z$ ) with cold absorption at $z=0$, also gives an acceptable fit (Table 1) with an absorbing column density of only $2.7_{-1.6}^{+4.5} \times 10^{20}$ atoms $\mathrm{cm}^{-2}$, close to and consistent with the Galactic value (Fig. $3 b$ ). Fixing $N_{\mathrm{H}}$ to the Galactic values gives a better costrained temperature for the blackbody with $k T=0.68_{-0.03}^{+0.04}$ (rest frame, Table 1). An optically thin thermal plasma model (Raymond \& Smith 1977) at $z=1.049$ (with abundances fixed at 0.3 solar, the mean value found in low-redshift clusters of galaxies, e.g., Hatsukade 1989) also gives an acceptable fit but still requires intrinsic absorption at a level comparable with a power law (Table 1). A Raymond-Smith plasma at $z=0$ and absorption fixed at the Galactic value gives an unacceptable fit (Table 1).

Another means of making a spectrum that peaks around 1 $\mathrm{keV}$ is to invoke strong line emission. A fit to a single broad

TABLE 1

SPECTRAL Fits to the ROSAT PSPC SPECtRum OF 3C 212

\begin{tabular}{|c|c|c|c|c|c|}
\hline Model Fitted & $\alpha_{E}$ or $k T^{\mathrm{a}, \mathrm{b}}$ & $N_{\mathbf{H}}(\text { free })^{\mathbf{a}, \mathbf{c}}$ & Normalization $^{\mathrm{a}, \mathrm{d}}$ & $\chi^{2}$ (dof) & $P\left(>\chi^{2}\right)$ \\
\hline \multicolumn{6}{|l|}{ Power-Law: } \\
\hline$+N_{\mathrm{H}}(z=0$, free $) \ldots \ldots$ & $1.9_{-1.0}^{+1.0}$ & $\left(3.2_{-1.6}^{+1.8}\right) \times 10^{21}$ & $4.1_{-2.0}^{+2.7}$ & $14.4(21)$ & 0.852 \\
\hline$+N_{\mathrm{H}}(\text { Galactic, fixed })^{\mathrm{e}} \ldots \ldots \ldots \ldots \ldots \ldots \ldots \ldots$ & $0.14_{-0.09}^{+0.09}$ & & $1.73_{-0.07}^{+0.07}$ & $32.0(22)$ & 0.077 \\
\hline $\begin{array}{l}+N_{\mathrm{H}}(\text { Galactic, fixed })^{\mathrm{e}} \\
\quad+N_{\mathrm{H}}(z=1.049, \text { free })\end{array}$ & $1.4_{-0.6}^{+0.8}$ & $\left(8.7_{-6.3}^{+7.5}\right) \times 10^{21}$ & $3.0_{-0.9}^{+1.4}$ & $15.2(21)$ & 0.813 \\
\hline $\begin{array}{l}\text { Blackbody: } \\
\quad(z=1.049)+N_{\mathrm{H}}(z=0, \text { free }) \ldots \ldots \ldots \ldots . .\end{array}$ & $0.70_{-0.07}^{+0.07}$ & $\left(2.7_{-1.6}^{+4.5}\right) \times 10^{20}$ & $2.5_{-0.7}^{+1.3}$ & $13.1(21)$ & 0.805 \\
\hline$(z=1.049)+N_{\mathbf{H}}(\text { Galactic, fixed })^{\mathrm{e}} \ldots \ldots \ldots$ & $0.68_{-0.03}^{+0.04}$ & & $2.6_{-0.3}^{+0.2}$ & $13.4(22)$ & 0.894 \\
\hline \multicolumn{5}{|l|}{ Optically Thin Thermal (Raymond-Smith):f } & 0.890 \\
\hline$(z=0)+N_{\mathrm{H}}($ Galactic, fixed $) \ldots \ldots \ldots \ldots \ldots$ & $<80$ & & $1.3_{-0.4}^{+0.1}$ & $31.2(22)$ & 0.092 \\
\hline $\begin{aligned}(z & =1.049)+N_{\mathrm{H}}(\text { Galactic, fixed })^{\mathrm{e}} \ldots \ldots \ldots \\
& +N_{\mathrm{H}}(z=1.049, \text { free })\end{aligned}$ & $3.0_{-1.3}^{+7.0}$ & $\left(5.8_{-4.4}^{+4.7}\right) \times 10^{21}$ & $3.8_{-1.3}^{+2.5}$ & $15.3(21)$ & 0.808 \\
\hline
\end{tabular}

${ }^{\text {a }}$ Errors are $68 \%$ for two interesting parameters when $N_{\mathrm{H}}$ is free, and for one interesting parameter when $N_{\mathrm{H}}$ is fixed.

${ }^{\mathrm{b}} \alpha_{E}$ is the energy spectral index $\left(f=\right.$ Norm. $\left.E^{-\alpha_{E}}\right)$; temperatures are in $\mathrm{keV}$.

c Atoms $\mathrm{cm}^{-2}$. Morrison \& McCammon 1983 cross sections were used.

d Normalization units are: for power-law fits: $10^{-4} \mathrm{keV} \mathrm{cm}^{-2} \mathrm{~s}^{-1} \mathrm{keV}^{-1}$ at $1 \mathrm{keV}$; for blackbody fits: $10^{-4} \mathrm{keV} \mathrm{cm}^{-2} \mathrm{~s}^{-1}$ at $k T$

$\mathrm{keV}$; for optically thin thermal fits: $10^{-17} \mathrm{~cm}^{-5}\left(\left[\int n_{e}^{2} d V\right] /\left[4 \pi D_{L}^{2}\right]\right)$.

' With $N_{\mathrm{H}}$ (Galactic) fixed at $3.7 \times 10^{20}$ atoms $\mathrm{cm}^{-2}$.

f With abundances fixed at 0.3 solar. 

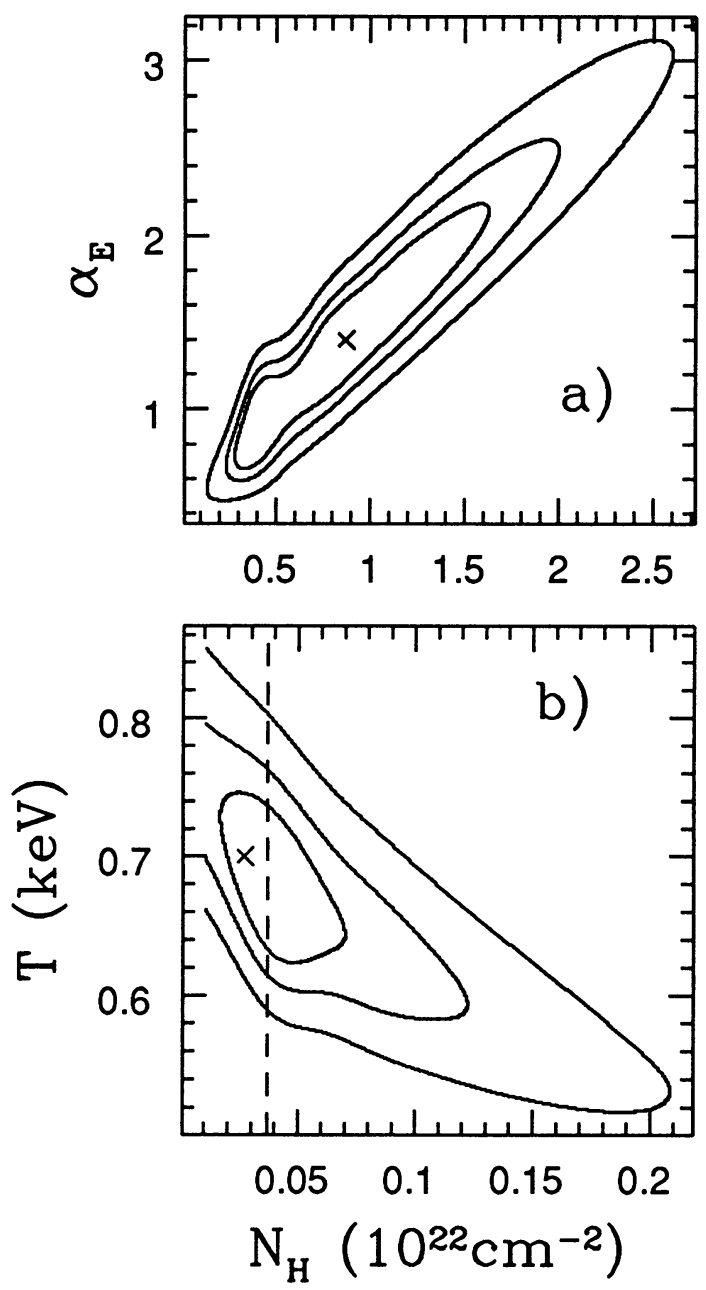

FIG. 3.-Contours of allowed values of $(a)$ energy spectral index and $z=1.049$ column density, assuming Galactic absorption: $(b)$ blackbody temperature (at $z=1.049$ ) and column density at $z=0$. Crosses mark the best-fit values for each model. The Galactic $\mathrm{H}$ I column density is shown in $(b)$ as a dashed line.

Gaussian line does give a good fit to the spectrum, with a line energy of $0.9 \pm 0.1 \mathrm{keV}$ (observed), and a sigma of $0.5 \pm 0.1$ $\mathrm{keV}$ (observed), that is, $0.55 \mathrm{c}$. A narrow line gives an unacceptable $\chi^{2}$. There is no obvious candidate for such a line at $z=1$. The strong iron $\mathrm{L}$ line complex at $\sim 1 \mathrm{keV}$ would be the obvious candidate, but at $z=1$ this complex is redshifted into the $0.5 \mathrm{keV}$ "hole" in the PSPC response (due to carbon absorption in the detector window, ROSAT Mission Description 1991).

Fluxes and luminosities for the best-fit models are given in Table 2 . We include the (0.4-4) keV luminosity since this is the observed band in the $z=1$ rest frame.

\section{DISCUSSION}

We have two contrasting explanations for the lack of lowenergy photons in the ROSAT X-ray spectrum of 3C 212: an absorbed power law, or an unabsorbed blackbody. A blackbody would be an unprecedented spectral shape, but it cannot be dismissed. Both the absorbed power law and the blackbody model can explain the lack of optical photons.

For a power-law fit, the large X-ray column density found toward 3C 212 is immediately interpretable as the cause of the

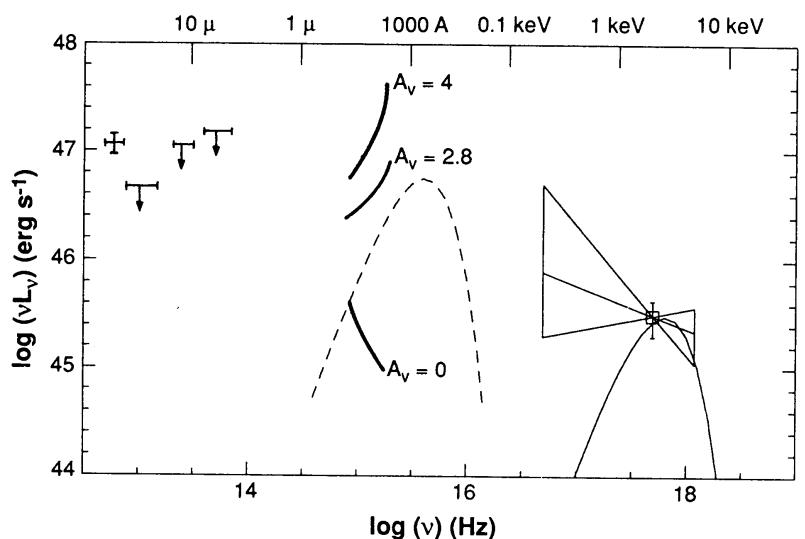

FIG. 4.-Rest frame spectral energy distribution for 3C 212 from radio to $\mathrm{X}$-ray. The power-law fit to the ROSAT X-ray spectrum is shown as a "bowtie" that shows the $68 \%$ confidence limits on the slope. The blackbody fit to the X-ray spectrum is shown as the solid curve. Since the power-law slope is shown corrected for the implied absorption it does not overlap the blackbody fit below about $2 \mathrm{keV}$. The optical continuum data are from Smith \& Spinrad (1980) and are shown as observed $\left(A_{V}=0\right)$, dereddened to match a typical quasar $\left(A_{V}=2.8\right)$, and dereddened $\left(A_{V}=4\right)$ to match the best-fit column density found from the $\mathrm{X}$-ray spectrum (assuming a power law and standard Milky Way dust-to-gas conversions; see text). A blackbody of $T=5 \times 10^{4} \mathrm{~K}$ is shown to represent the typical quasar UV bump, for comparison. The IRAS upper limits (Impey \& Neugebauer 1988) are also shown. The $100 \mu \mathrm{m}$ point may be due to cirrus.

red continuum found in the optical/ultraviolet by Smith \& Spinrad (1980). Similar amounts of absorption are common in Seyfert galaxies (Turner \& Pounds 1989) and give reasonable quantitative results when applied to the optical spectrum of $3 \mathrm{C} 212$. The observed optical continuum can be dereddened to give the characteristic "UV bump" shape seen in the normal quasars with $A_{V}=2.8$ (Fig. 4), assuming standard dust-to-gas ratios and Milky Way dust (Jenkins \& Savage 1974). An $N_{\mathrm{H}}$ of $10^{22}$ atoms $\mathrm{cm}^{-2}$ implies $A_{V}=4 \mathrm{mag}$, assuming the same conversions, and so overcorrects the slope of the ultraviolet (emitted) continuum in 3C 212 from $+3.6 \pm 0.3$ (in $f_{v}$, Smith \& Spinrad 1980) to -6.4 , well above that of a normal quasar such as $3 \mathrm{C} 273$ (Fig. 4). ${ }^{2}$ This is not a particularly strong difficulty for this model. Even within the Galaxy the dust-to-gas ratio varies by factors of 1.5 (Savage \& Mathis 1979). In Seyfert galaxies while $A_{V}$ and X-ray $N_{\mathrm{H}}$ are correlated, there is always an offset in the sense of seeing less $A_{V}$ than implied by simple application of Galactic conversion values to the X-ray-derived $N_{\text {H }}$ (e.g., Turner \& Pounds 1989). The optical/ultraviolet spectrum of 3C 212 shows no $\lambda 2175$ feature to a level of $A_{V} \sim 0.5$ (Smith \& Spinrad 1980). For Galactic-type dust at the column densities implied by the X-ray spectrum such a feature would be extremely obvious (Smith \& Spinrad 1980). So the dust causing the reddening toward 3C 212 must either be unlike that of the Milky Way, or be intervening with $z<0.4$, so that the $\lambda 2175$ feature is not observable from the ground. In the power-law case the $2500 \AA$ to $2 \mathrm{keV}$ dereddened index, $\alpha_{\text {ox }}$, is about 1.4 , similar to the mean $\alpha_{\text {ox }}$ in radio-loud quasars. Aldcroft, Bechtold, \& Elvis (1994) report Mg II absorption lines in $3 \mathrm{C} 212$ close to the emission redshift. The lines appear strong, but because of saturation and the strong possibility of multiple

\footnotetext{
${ }^{2}\left[E(3000-2000 \AA)=0.92 A_{V}, \Delta \alpha(3000-2000 \AA)=2.5 A_{V}.\right]$
} 
components no good estimate of the implied column density can be given. Nevertheless, low-ionization absorbing material is seen to be present in 3C 212, which supports the absorbed power-law model. In summary reddening can reasonably explain all the optical and X-ray peculiarities of 3C 212.

The blackbody fit is also readily interpreted as a "UV bump" that has a characteristic temperature some 100 times that of a normal UV bump $\left(\sim 8 \times 10^{6} \mathrm{~K}\right.$ rather than $\left.\sim 5 \times 10^{4} \mathrm{~K}\right)$. The extrapolation of the Rayleigh-Jeans tail of the blackbody falls well below the observed optical continuum (Fig. 4). In the blackbody case $\alpha_{\text {ox }} \sim 0.85$, which is among the smallest known values, even in radio-loud quasars. The blackbody model implies an emitting area of $1.4 \times 10^{22} \mathrm{~cm}^{-2}$, and a radius of $3.3 \times 10^{10} \mathrm{~cm}$. If we assume that the radius corresponds to 10 Schwarzchild radii then the corresponding black hole mass would be $\sim 10^{4} M_{\odot}$ and the emission highly superEddington. The emission must then come from larger radii.

The two models have extreme differences in the implied shape of their ionizing UV to soft X-ray continuum, with a factor of $\sim 10^{3}$ difference in the number of ionizing photons (Table 3). They might be expected to predict quite different emission-line ratios. To investigate these differences as a means of distinguishing between the two models we calculated, with the photoionization code CLOUDY (Ferland 1991), the expected line intensities and equivalent widths in both cases. For the power-law case we assumed the best-fit X-ray power law and an optical spectrum dereddened by $A_{V}=2.8$. For blackbody case we assumed the observed optical continuum and extrapolated it into the UV. We considered two typical values of the ionization parameter $U\left(10^{-2}\right.$ and $\left.10^{-3}\right)$, and a covering factor $f_{C}=0.1$. The results are shown in Table 3 .

We compare these with the line strengths from Smith \& Spinrad (1980) in Table 3. We note that these equivalent widths are uncertain. Aldcroft et al. find $\mathrm{EW}(\mathrm{Mg} \mathrm{II})_{\text {rest }}=31 \pm 2 \AA$, about double the value in Smith and Spinrad. (Aldcroft et al. do not cover $\mathrm{C} \mathrm{III]}$.) In spite of the huge differences between the assumed ionizing continua, the photoionization predictions provide no convincing discriminants between the models given the observed quantities. The similarity of the predictions is partly because the increased number of ionizing photons in the dereddened power law is almost exactly matched by the

TABLE 3

Photoionization MODELS

\begin{tabular}{|c|c|c|c|c|}
\hline Model & \multicolumn{2}{|c|}{ Power Law ${ }^{a}$} & \multicolumn{2}{|c|}{ Blackbody } \\
\hline EW (Mg II) observed . & \multicolumn{2}{|c|}{$11-18.3$} & \multicolumn{2}{|c|}{$11-18.3$} \\
\hline $\mathrm{EW}(\mathrm{C}$ III $])$ observed $\ldots \ldots \ldots \ldots \ldots \ldots$ & \multicolumn{2}{|c|}{$2.2-6.6$} & \multicolumn{2}{|c|}{$2.2-6.6$} \\
\hline $\mathrm{Mg}$ II/C III $]$ observed $\ldots \ldots \ldots \ldots \ldots \ldots$ & \multicolumn{2}{|c|}{$4.9 \pm 2.8$} & \multicolumn{2}{|c|}{$7 \pm 4$} \\
\hline Number of ionizing photons ......... & \multicolumn{2}{|c|}{$2 \times 10^{57}$} & \multicolumn{2}{|c|}{$4 \times 10^{54}$} \\
\hline Ionization parameter ............... & $10^{-2}$ & $10^{-3}$ & $10^{-2}$ & $10^{-3}$ \\
\hline $\mathrm{EW}(\mathrm{Mg} \mathrm{II})^{\mathrm{b}}$ & 28 & 70 & 35 & 9 \\
\hline $\mathrm{EW}(\mathrm{C}$ III $])^{\mathrm{b}} \ldots$ & 34 & 18 & 23 & 0.5 \\
\hline $\mathrm{Mg}$ II $/ \mathrm{C}$ III $] \ldots .$. & 0.44 & 2.4 & 2.7 & 31 \\
\hline $\mathbf{H} \alpha / \mathbf{H} \beta \ldots \ldots \ldots$ & 6.4 & 6.4 & 3 & 22 \\
\hline Radius $(\mathrm{pc})^{\mathrm{c}} \ldots$ & 7.6 & 23 & 0.3 & 1 \\
\hline He II $1640 / \mathbf{H} \beta \ldots$ & 1.72 & 1.33 & 0.06 & 0.09 \\
\hline 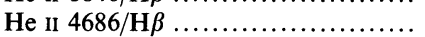 & 0.185 & 0.14 & 0.007 & 0.01 \\
\hline
\end{tabular}

a Observed values are corrected for reddening by $A_{V}=2.8$, assuming a standard Milky Way reddening law (Savage \& Mathis 1979).

${ }^{b}$ For $10 \%$ covering factor.

c Assuming a gas density of $10^{9} \mathrm{~cm}^{-3}$ increased number of emitted line photons, since both lines and continuum are dereddened by the same amount. Another limitation is that there are few observed lines to provide constraints. Only Mg II $\lambda 2798$ and C III] $\lambda 1909$ are clearly detected (Smith \& Spinrad 1980). All the hydrogen lines lie outside the observed range (1500-3560 $\AA$, rest). (Because of the poor signal-to-noise ratio, it is possible that the observed C III] 1909 emission is actually dominated by a narrow component, in which case the $\mathrm{Mg} \mathrm{II} / \mathrm{C} \mathrm{III]}$ line ratio is an upper limit.) Better diagnostics could be searched for. For example, the greatest difference between the two continua is in the EUV, so that the He II lines, which require $\lambda<304 \AA$ photons for their production, should provide a clean discriminant between the two models (Table 3 ).

None of the above arguments allows us to choose clearly between the two possibilities. However, the blackbody case has never before been observed in any AGN, whereas reddening by similar amounts is common. We shall assume, using Occam's razor, that obscuration is the cause of the X-ray cutoff and of the red optical continuum in the rest of the paper. The simplest means of discriminating between the two cases would be an observation at higher X-ray energies $(E>2 \mathrm{keV})$, where the shapes of the two continua differ strongly. Such an observation is possible with the instruments on board ASCA.

\subsection{Thermal Emission}

Since an absorbed optically thin plasma also gives an acceptable fit to the $3 \mathrm{C} 212 \mathrm{X}$-ray spectrum we need to consider possible origins of this type. A cluster of galaxies around the quasar is one possibility.

Extended, thermal emission has been detected around a number of nearby radio galaxies (Worrall \& Birkinshaw 1994), but has a maximum luminosity of $\sim 10^{42} \mathrm{ergs} \mathrm{s}^{-1}$. In contrast, the implied X-ray luminosity of 3C 212 would place it among the highest found in clusters of galaxies at low redshift (e.g., A2163 at $6.4 \times 10^{45}$ ergs s $^{-1}$, Arnaud et al. 1992). Clusters seem to undergo significant negative evolution by $z=0.5$ (i.e., they become fainter at higher $z$, Henry et al. 1991), so by $z=1$ clusters of the luminosity of 3C 212 are likely to be extremely rare. Crawford \& Fabian (1993) have claimed that the PSPC image of the $z=1.079$ radio galaxy 3C 356 shows extended $\mathrm{X}$-ray emission with a luminosity of $\sim 2.5 \times 10^{44} \mathrm{ergs} \mathrm{s}^{-1}$, although this result must be treated with caution since it is based on only 32 net counts, and because source confusion must be a problem for a source some 20 times fainter than $3 \mathrm{C} 212$.

A direct test can be derived from the diameter of the $3 \mathrm{C} 212$ $\mathrm{X}$-ray source. A limit on the source size can be obtained by convolving Gaussian functions of increasing width with the 3C 273 radial photon distribution, and comparing them with the observed distribution. For 3C 212 this method gives a limit of $\lesssim 8^{\prime \prime}(\lesssim 0.07 \mathrm{Mpc}$ at $z=1.049)$ on the width of the Gaussian. This is much smaller than the typical core radii of clusters which are of order $0.2-0.5 \mathrm{Mpc}\left(23^{\prime \prime}-57^{\prime \prime}\right)$.

Photoionization modeling with CLOUDY provides another argument against an extended X-ray source. Extrapolating the observed optical spectrum into the ultraviolet, without an additional X-ray component, produces line intensities that, for any reasonable value of $U$ and $f_{\mathrm{C}}$, are an order of magnitude weaker than observed (EW Mg II $=0.38 \AA$ ).

The "Raymond" luminosities in Table 2 should thus be taken as upper limits to a cluster contribution. A substantial contribution from a cluster component is not excluded by our 
$\mathrm{X}$-ray data. The relative strength of a thermal extended component could be constrained by a high-resolution X-ray image (e.g., with the ROSAT HRI).

A thermal plasma in the quasar nucleus could exist, confined in small dense clouds as in the models of Barvainis (1993), but at temperatures some 100 times higher than he proposed. A high-resolution X-ray spectrum is needed to put limits on the line emission expected in this model.

\subsection{A Power Law and Possible Absorption Sites}

Lower luminosity analogues of 3C 212 are well known. Absorption at a similar level in Seyfert galaxies (Turner \& Pounds 1989) has already been mentioned. In addition, several $3 \mathrm{C}$ radio galaxies have optical and near-infrared slopes similar to those of 3C 212 (Elvis et al. 1984). At least two of these vary by a factor $50 \%$ in 3 years in the infrared indicating that the steepness is not that of a host galaxy starlight spectrum. Strong, broad Paschen- $\alpha$ has been seen in some radio galaxies (Carleton et al. 1984), implying reddening of the spectrum. Two of the red radio galaxies studied by Elvis et al. (1984) show signs of strong X-ray absorption: the Ginga spectrum of $3 \mathrm{C} 445$ indicates an absorbing column density of $1.7 \times 10^{23}$ atoms $\mathrm{cm}^{-2}$ (Pounds 1990). Similarly, 3C 109 has a ROSAT PSPC spectrum with few low-energy counts that Allen \& Fabian (1992) interpret as X-ray absorption of $\sim 2 \times 10^{21}$ atoms $\mathrm{cm}^{-2}$. Another radio galaxy, NGC 6251, has a PSPC spectrum that may show an absorbed power law (Birkinshaw \& Worrall 1993), but in this case a thermal explanation is also tenable, because the low redshift of the galaxy allows the Fe-L lines to simulate the appearance of a low-energy cutoff. 3C 109 is only a factor of 4 less luminous than 3 C 212 in X-rays, and so only a minor extension of known source properties is needed to include 3C 212 as an obscured AGN.

These obscured radio galaxies and 3C 212 each have X-ray luminosities that are a factor 10-100 times higher than those of radio-quiet $\mathrm{AGNs}$ showing X-ray absorption (Lawrence \& Elvis 1982; Turner \& Pounds 1989). Is there some property associated with radio emission that allows high-luminosity AGNs to exhibit strong obscuration? Radio-loud AGNs may have an additional site for absorption unavailable to radioquite AGNs. Since $3 \mathrm{C}$ radio galaxies lie almost exclusively in elliptical galaxies, some property of the host galaxy may be responsible. Alternatively a selection effect may be at work. The large physical size of the radio lobes may allow the recognition of activity in AGNs whose radio-quiet counterparts would be inconspicuous.

At $z=1.049$ 3C 212 lies at a considerably longer lookback time and path length than the unabsorbed high-luminosity AGNs previously studied in X-rays. This opens up the possibilities of (1) evolution in the AGN environment and (2) intervening absorbers.

1. 3C 212 may well lie in a cluster of galaxies. Radio-loud quasars at $z>0.5$ are often found in rich clusters (Yee \& Green 1987; Ellingson, Yee, \& Green 1991; Yee \& Ellingson 1993). An analogy with Cygnus $A$ is informative. 3C 212 is similar to Cignus A in its FRII radio morphology (Akujor et al. 1991), physical size (core-hot spot separation of $50 \mathrm{kpc}$, Akujor et al. 1991 ; cf. $100 \mathrm{kpc}$ for Cygnus A, Carilli et al. 1991), presence of obscured X-ray $\left(N_{\mathrm{H}} \sim 8 \times 10^{22}\right.$ atoms $\mathrm{cm}^{-2}$ Arnaud et al. 1987) and optical (Djorgovski et al. 1991) nuclei, and radio luminosity (Becker, White, \& Edwards 1991) - but 3C 212 is 50 times more X-ray luminous. Cygnus $\mathrm{A}$ lies in a cluster with a strong cooling flow (Arnaud et al. 1984). Elvis et al. (1994) have discussed cooling flows around host elliptical galaxies as a possible source of the obscuration they find toward several $z=3$ quasars. Cluster cooling flows can contain column densities of cool X-ray-absorbing material up to a few $\times 10^{21}$ atoms $\mathrm{cm}^{-2}$ (White et al. 1991). Such absorbers could well account for the $\sim 15 \%$ of $3 \mathrm{C}$ quasars that Smith \& Spinrad (1980) find to have red optical/ultraviolet continua.

2. Intervening absorption line systems may also have sufficient space density to produce obscuration in a significant fraction of objects by $z=1$. Absorption line systems have not been studied toward 3C 212 , because of its faint optical/ultraviolet continuum. A statistical argument can be made instead. The high column density "damped Lyman- $\alpha$ systems" are the obvious absorber candidates. A neutral fraction of 0.01 is possible in damped Lyman- $\alpha$ systems (Elvis et al. 1994; Lanzetta et al. 1991) so a system with $N(\mathrm{H} \mathrm{I})=10^{20}$ atoms $\mathrm{cm}^{-2}$ could produce the observed X-ray absorption, assuming it has solar abundances. Lanzetta et al. (1991) give a $\log N\left(>N_{\mathrm{H}}\right) / \log \left(N_{\mathrm{H}}\right)$ relation for Lyman- $\alpha$ absorbers which gives an expected number of absorbers with $N(\mathrm{H} I)>2 \times 10^{20}$ along a given line of sight to $z=1$ of 0.06 or 0.16 depending on the choice of parameters. This is similar to the fraction of red quasars in the 3C sample (Smith \& Spinrad 1980). Primordial material still produces X-ray absorption but would need a hydrogen column density some 5 times larger (Morrison \& McCammon 1983; Elvis et al. 1994) so reducing the number of absorbers available by a factor $\sim 15$. Since damped Lyman- $\alpha$ systems have not been studied at $z<1.5$ and strong evolution to lower densities at lower redshifts is expected in some models (Wolfe 1988), the expected number of $0.06-0.16$ is an upper limit.

Compared with the high-redshift quasars studied by Elvis et al. (1994) it is striking that only 3C 212 shows strong reddening in the optical/ultraviolet continuum. This suggests a different origin may apply to $3 \mathrm{C} 212$. A third possibility is obscuration by a nuclear torus, as much discussed recently for low-redshift AGNs (e.g., Pier \& Krolik 1992a, b). 3C 212 is not a strongly lobe-ciominated radio source. At $6 \mathrm{~cm}$ (observed) $3 \mathrm{C} 212$ has a core flux of $186 \mathrm{mJy}$ (Akujor et al. 1991) and a total flux of $746 \pm 102 \mathrm{mJy}$ (Gregory \& Condon 1991). The ratio of radio emission in the nucleus compared to the lobes, $R=f$ (core)/ $f$ (extended), is thus 0.33 in 3 C 212. For typical lobe and core spectral indices, $R$ would be smaller by about a factor 2 in the rest frame at $6 \mathrm{~cm}$. This compares with values $0.1-0.01$ for typical lobe-dominated objects (Orr \& Browne 1982). So 3C 212 is marginally lobe-dominated and may be viewed at quite a high inclination.

\section{CONCLUSIONS}

The ROSAT X-ray spectrum of the red quasar $3 \mathrm{C} 212$ can be well fitted with a power law and an X-ray column density of $\sim 10^{22}$ atoms $\mathrm{cm}^{-2}$ (in the quasar frame). Obscuration by this material provides a natural explanation for the steep optical/ ultraviolet spectrum of this and similar quasars. The dereddened spectrum is similar to that of normal quasars. Since no $\lambda 2175$ absorption feature is seen, the dust responsible for the reddening must either be of non-Milky Way composition, or be intervening at a redshift that makes the $\lambda 2175$ feature unobservable from the ground.

Possible sites for obscuration include the AGN nucleus (such as a torus), intracluster cold material around the quasar (such as that found in low-redshift cooling flows), and an intervening damped Lyman- $\alpha$ system. 
A definitive discrimination between the absorbed power-law and unabsorbed blackbody models for the X-ray emission can easily be achieved with an ASCA observation, since the shape of the two emission components above $2 \mathrm{keV}$ is extremely different.

We thank our colleagues J. McDowell, A. Siemiginowska, T. Aldcroft, and the anonymous referee for useful comments, and M. Birkinshaw for a careful reading of the manuscript. This research has made use of the NASA/IPAC Extragalactic Database (NED) which is operated by the Jet Propulsion Laboratory, California Institute of Technology, under contract with the National Aeronautics and Space Administration. This work was supported by NASA grants NAGW-2201 (LTSARP), NAG 5-1872 and NAG 5-1536 (ROSAT), and NAS 8-39073 (ASC), and NASA contracts NAS 5-30934 (RSDC), NAS 5-30751 (HEAO 2).

\section{REFERENCES}

Akujor, C. E., Spencer, R. E., Zhang, F. J., Davis, R. J., Browne, I. W. A., \& Fanti, C. 1991, MNRAS, 250, 214

Aldcroft, T. L., Bechtold, J., \& Elvis, M. 1994, AJ, in press

Allen, S. W., \& Fabian, A. C. 1992, MNRAS, 258, 29P

Arnaud, K. A., Fabian, A. C., Eales, S. A., Jones, C., \& Forman, W. 1984, MNRAS, 211, 981

Arnaud, K. A., Johnstone, R. M., Fabian, A. C., Crawford, C. S., Nulsen, P. E. J., Shafer, R. A., \& Mushotzky, R. F. 1987, MNRAS, 227, 241

Arnaud, M., Hughes, J. P., Forman, W., Jones, C., Lachieze-Rey, M., Yamashita, K., \& Hatsukade, I. 1992, ApJ, 390, 345

Barvainis, R. 1993, ApJ, 412, 513

Becker, R. H. White, R. L. \& Edwards, A. L. 1991, ApJS, 75,

Birkinshaw, M., \& Worrall, D. M. 1993, ApJ, 412, 568

Bregman, J. N., Glassgold, A. E., Huggins, P. J., \& Kinney, A. L. 1985, ApJ, 291,505

Bregman, J. N., Lebofsky, M. J., Aller, M. F., Rieke, G. H., Aller, H. D., Hodge, P. E., Glassgold, A. E., \& Huggins, P. J. 1981, Nature, 293, 714

Browne, I. W. A., \& Murphy, D. W. 1987, MNRAS, 226, 601

Carilli, C. L., Perley, R. A., Dreher, J. W., \& Leahy, J. P. 1991, ApJ, 383, 554

Carleton, N. P., Willner, S. P., Rudy, R. J., \& Tokunaga, A. T. 1984, ApJ, 284,

Crawford, C. S., \& Fabian, A. C. 1993, MNRAS, 260, L15

Djorgovski, S., Weir, N., Matthews, K., \& Graham, J. R. 1991, ApJ, 372, L67

Done, C., \& Fabian, A. C. 1989, MNRAS, 240, 81

Ellingson, E., Yee, H. K., \& Green, R. F. 1991, ApJ, 371, 49

Elvis, M., Fiore, F., Wilkes, B. J., McDowell, J., \& Bechtold, J. 1994, ApJ, 422, 00

Elvis, M., Green, R. F., Bechtold, J., Schmidt, M., Neugebauer, G., Soifer, B. T., Matthews, K., \& Fabbiano, G. 1986, ApJ, 310, 291

Elvis, M., Willner, S. P., Fabbiano, G., Carleton, N. P., Lawrence, A., \& Ward, M. J. 1984, ApJ, 280, 574

Eracleous, M., \& Halpern, J. P. 1994, ApJS, 90,

Ferland, G. 1991, 'HAZY', OSU Astronomy Department Internal Report

Gregory, P. C., \& Condon, J. J. 1991, ApJS, 75, 1011

Hatsukade, I. 1989, Ph.D. thesis, ISAS Tokyo

Henry, J. P., Gioia, I. M., Maccacaro, T., Morris, S. L., Stocke, J. T., \& Wolter, A. 1991, ApJ, 386, 408

Impey, C., Lawrence, C. R., \& Tapia, S. 1991, ApJ, 375, 46
Impey, C.. \& Neugebauer, G. 1988, AJ, 95, 307

Jenkins, E. B., \& Savage, B. D. 1974, ApJ, 187, 243

Krolik, J. H., Horne, K., Kallman, T. R., Malkan, M. A., Edelson, R. A., \& Kriss, G. A. 1991, ApJ, 371, 541

Lanzetta, K. M., Wolfe, A. M., Turnshek, D. A., Lu, L., McMahon, R. G., \& Hazard, C. 1991, ApJS, 77, 1

Lawrence, A., \& Elvis, M. 1982, ApJ, 256, 410

Moore, R. L., \& Stockman, H. S. 1984, ApJ, 279, 465

Morrison, R., \& McCammon, D. 1983, ApJ, 270, 119

Orr, J. B., \& Browne, I. W. A. 1982, MNRAS, 200, 1067

Pfefferman, E., et al. 1987, Proc. SPIE, 733, 519

Pier, E. A., \& Krolik, J. H. 1992a, ApJ, 399, L23 $1992 \mathrm{~b}$, ApJ, 401, 99

Pounds, K. A. 1990, MNRAS, 242, 2P

Raymond, J., \& Smith, B. W. 1977, ApJS, 35, 419

Rieke, G. H., Lebofsky, M. J., \& Kinman, T. D. 1979, ApJ, 232, L151

Rieke, G. H., Lebofsky, M. J., \& Wisniewski, W. Z. 1982, ApJ, 263, 73

ROSAT Mission Description. 1991, NASA NRA 91-OSSA-3, Appendix F

Savage, B. D., \& Mathis, J. S. 1979, ARA\&A, 17, 73

Smith, H. E., \& Spinrad, H. 1980, ApJ, 236, 419

Stark, A. A., Gammie, C. F., Wilson, R. W., Bally, J., Linke, R., Heiles, C., \& Hurwitz, M. 1992, ApJS, 79, 77

Sun, W.-H., \& Malkan, M. A. 1989, ApJ, 346, 68

Svensson, R. 1990, in Physical Processes in Hot Cosmic Plasmas, ed. W. Brinkman (Dordrecht: Kluwer)

Trümper, J. 1983, Adv. Space Res., 2, 4, 241

Turner, T. J., \& George, I. M. 1992, ROSAT PSPC Calibration Guide, GSFC OGIP Report

Turner, T. J., \& Pounds, K. A. 1989, MNRAS, 240, 833

White, D. A., Fabian, A. C., Johnstone, R. M., Mushotzky, R. F., \& Arnaud, K. A. 1991, MNRAS, 252, 72

Wilkes, B. J. 1986, MNRAS, 218, 331

Wolfe, A. M. 1988, in QSO Absorption Lines, ed. J. C. Blades, D. Turnshek, \& C. A. Norman (Cambridge: Cambridge Univ. Press), 297

Worrall, D. M., \& Birkinshaw, M. 1994, ApJ, in press

Yee, H. K., \& Ellingson, E. 1993, ApJ, 411, 43

Yee, H. K., \& Green, R. F. 1987, ApJ, 319, 28

Zdziarski, A. A., \& Lamb, D. Q. 1986, ApJ, 305, 45 